\title{
基于刚性平面布局的折痕设计
}

\author{
孙晓鹏 1,3 )，金美辰 ${ }^{1,2)}$ ，李姣姣1)，王振燕1) \\ ${ }^{1)}$ (辽宁师范大学计算机与信息技术学院计算机系统研究所 大连 116029) \\ 2) (大连财经学院管理学院 大连 116622) \\ 3) (智能通信软件与多媒体北京市重点实验室(北京邮电大学) 北京 100876) \\ (cadcg2008@hotmail.com)
}

\begin{abstract}
摘 要: 折叠是近年来柔性机器人领域的研究热点之一. 在折痕设计方法中, 针对平面布局误差大、分布不均和折痕 复杂等问题，提出基于刚性平面布局的折痕设计方法，可在无剪切、拉伸和粘贴的约束下，实现三角网格模型的顶点 折痕和边折痕设计. 首先, 基于 ARAP 刚性局部参数化拆解三角网格模型, 并以最优旋转和平移变换实现刚性平面 布局, 将三角网格模型拆分为参数平面空间内均匀分布、互不相连的三角面片; 然后, 构造边折痕, 并基于增量法 Voronoi 和站点约束篮选有效站点, 并以其构造顶点折痕; 最后, 将局部折痕嵌人平面布局, 实现三角网格模型完整 的折痕设计. 在相同环境下对 LSCM、均值参数化方法以及文中方法的平面布局误差值进行比对分析，同时对平面布 局过程中的能量值, 以及平面布局结果的有效值进行分析. 结果表明, 文中方法平面布局误差较低, 稳定性较高, 三 角面片空间分布较为均匀, 适用于较大规模的三角网格模型.
\end{abstract}

关键词：折痕设计；平面布局; Voronoi 站点; 边折痕; 顶点折痕

中图法分类号: TP391.41 DOI: 10.3724/SP.J.1089.2021.18312

\section{Crease Design Based on Rigid Layout}

\author{
Sun Xiaopeng ${ }^{1,3)}$, Jin Meichen ${ }^{1,2)}$, Li Jiaojiao ${ }^{1)}$, and Wang Zhenyan ${ }^{1)}$ \\ 1) (Computer Systems Research Institute, Department of Computer and Information Technology, Liaoning Normal University, Dalian 116029) \\ 2) (Department of Management, Dalian University of Finance and Economics, Dalian 116622) \\ 3) (Beijing Key Laboratory of Intelligent Telecommunications Software and Multimedia, Beijing University of Posts and Telecommunications, Beijing \\ 100876)
}

\begin{abstract}
Folding is one of the hotspots in the research of flexible robots in recent years, crease design based on rigid layout is proposed to solve the problems such as large error of plane layout, uneven distribution, and complex crease, and realizes vertex crease and edge crease design of 3D triangular mesh without cutting, stretching and pasting. Firstly, we disassemble the triangular mesh surface based on ARAP rigid local parameterization, and propose a rigid layout by optimizing the transformation of rotation and translation, to layout the triangular face of the mesh model into uniformly distribution without connection in the parametric plane space. Then, we construct the edge crease, and construct the selection of good sites using the incremental Voronoi method and the sites constraint, then construct the vertex creases using the good sites. Finally, we locate the local creases into the layout to complete the crease design of triangular mesh model. The layout error values of LSCM, the mean parametric method and proposed method are compared and analyzed; the energy value and the effective value in layout are analyzed in the same experimental environment. The results show that proposed method has the advantages of low error, high stability and uniform distribution, and is suitable for larger-scale triangular mesh.
\end{abstract}

收稿日期：2020-04-13; 修回日期：2020-10-15. 基金项目：国家自然科学基金(61472170). 孙晓鹏(1968一)，男，博士，教授，硕 士生导师, CCF 高级会员, 主要研究方向为计算机图形学; 金美辰(1994一), 女, 硕士, 主要研究方向为计算机图形学; 李姣姣 (1997-), 女, 硕士研究生, 主要研究方向为计算机图形学; 王振燕(1996-), 女, 硕士研究生, 主要研究方向为计算机图形学. 
Key words: crease design; layout; Voronoi sites; edge crease; vertex crease

将三角网格模型拆解并展开到平面，设计平 面折痕, 然后通过空间折叠还原逼近原始三维网 格模型, 是近年来的国际研究热点, 此需求首先来 自软体机器人设计等工业领域.

平面折痕设计过程一般包括三角网格模型自 三维空间向二维平面参数空间的映射 ${ }^{[1]}$ 、三角面片 在二维平面参数空间内旋转平移实现分离布局、在 分离的三角面片缝隙间插人边折痕 (edge-tucking mode, ETM) 和顶点折痕 (vertex-tucking mode, VTM) 等二维折痕单元 3 个关键步骤 ${ }^{[2]}$, 最终沿折 痕折叠, 实现自二维平面参数空间向原三角网格 模型外形的逼近. 其中主要困难在于三角网格模 型由三维空间映射到二维平面参数空间的过程中, 应尽可能地满足三角网格模型拉伸小、剪切少和三 角面片不变形等约束.

本文针对拉伸、剪切和变形等约束目标，以 $\mathrm{ARAP}$ (as rigid as possible)刚性局部参数化 ${ }^{[3]}$ 为预 处理，降低计算成本; 在平面布局阶段引人平移参 数, 实现三角面片均匀分布和无重叠布局; 最后定 义 ETM 和基于站点约束的 VTM 等二维折痕单元 ${ }^{[4]}$, 在二维平面参数空间内链接互相分离的三角面片, 实现三角网格模型的折痕设计.

\section{1 相关工作}

计算机折纸主要包含平面布局和空间折痕设 计 2 个部分工作, 其中平面布局可分为局部参数化 和旋转平移变换 2 步.

2010 年, $\mathrm{Tachi}^{[2]}$ 首次提出基于折缝分子的三 维网格模型折叠; 2013 年, $\mathrm{Tachi}^{[5]}$ 提出多约束条件 的折纸镶嵌，以避免面片之间的碰撞，2 种方法均 能够保留三维网格模型的基本形状特征. Zhu 等 ${ }^{[6]}$ 提出软折叠方法, 以交互方式提高效率, 可实现多 种复杂折叠形状，但模型可展性受限. 2017 年, Zhao 等 ${ }^{[7]}$ 设计一种弹出式卡片, 在二维平面设计 折痕并利用导电油墨印刷, 剪裁后可得到三维立 体模型. 同年, Miyashita 等 $^{[8]}$ 提出通过折叠 “外骨 骼” 实现机器变形，基于折纸思想设计折叠路径， 三维物体可在 2 种形态中转换. Zhao 等 ${ }^{[9]}$ 提出轴对 称三维折纸, 基于 Waterbomb 和 Yoshimura 设计轴 对称折痕, 折痕线相交于 1 点且折痕长度可变. 2019 年, $\mathrm{Li}$ 等 ${ }^{[10]}$ 提出由折纸“魔术球”和薄膜组成 的真空驱动夹具，控制伸缩即可抓取物体. 本文提
出的折痕设计方法在无碰撞的基础上满足了三维 模型的可展性.

近年来, 三维网格模型参数化方法已经能够 在很大程度上保留三维网格模型的基本特征, 但 依然无法保证模型每个三角面片的边长、夹角及面 积保持不变 ${ }^{[11-13]}$. 本文要求无误差还原三维网格 模型, 因此无法直接使用参数化方法, 仅以局部参 数化作为预处理. 2002 年, Lévy 等 ${ }^{[13]}$ 提出最小二乘 保角参数化(least squares conformal maps, LSCM) 方法, 最小化角度变形, 误差最小, 但对于存在非 边界奇异点的模型, 该方法无法准确找到边界. 2008 年 $\mathrm{Liu}$ 等 $^{[3]}$ 提出 Local/Global 局部参数化, 对 三维网格模型的所有三角面片单独进行局部参数 化, 网格三角面片只发生刚性变换, 然后全局缝合 为相互连接的二维平面图. 2013 年, Wang 等 ${ }^{[14]}$ 基 于静电理论提出均值参数化方法(MEAN)对开放 边界模型进行参数化, 该方法仅适用于亏格为 0 的 单边界模型, 无法处理有亏格的模型(如 Car 模型 亏格为 10), 且误差较大. 2018 年, Sawhney 等 ${ }^{[15]}$ 提 出边界优先平坦化, 预先给出目标参数化模型的 边长或曲率, 以保证参数化模型边界误差值达到 最小. 2018 年, 杜兴逸等 ${ }^{[16]}$ 提出基于场论实现质心 对齐的 Voronoi 划分, 固定各三角面片质心，基于 三角网格质心实现三角面片的平面布局, 由于未 考虑邻域信息，相邻三角面片布局后可能产生交 叉重叠. 2018 年, Liu等 ${ }^{[17]}$ 提出渐进参数化方法, 局 部进行刚性旋转, 可得到无误差的初步旋转模型. 同年, Soliman 等 ${ }^{[18]}$ 提出保角映射的最优雉奇点, 对每个面片单独计算可使其总面积失真最小. 本 文方法在局部参数化基础上完成平面布局设计, 完整保留三维模型的局部特征, 以最小误差逼近 原始三维网格模型.

目前，部分研究工作将三角面片之间的折痕

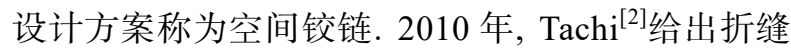
分子的定义, 可将互不相连的三角面片连接在一 起. 2015 年, Chen 等 ${ }^{[19]}$ 提出刚性厚板纸折叠方法, 其对称折叠方式适用于对称的边铰链, 但无法应 用在非对称模型上. 2016 年, Chen 等 ${ }^{[20]}$ 基于 Waterbomb 模式提出对称折叠动力学, 构造与折纸 等价的折叠动力学, 可保证点铰链折叠角度总和 为 $2 \pi$, 并避免折叠交叉. 2017 年, Guseinov 等 ${ }^{[21]}$ 提 出三维模型弯曲薄板设计方法, 在二维平面上生 成“片”布局作为铰链, 省略部分折叠, 但该方法对 
于折叠材料的要求很高, 保证弹性的同时要维持 平衡状态. $\mathrm{Li}$ 等 ${ }^{[22]}$ 提出基于折纸的流体驱动人工 肌肉, 将可折叠纸作为可伸缩骨架，通过流体介质 实现收缩弯曲等运动. 2019 年, Wang 等 ${ }^{[23]}$ 提出优 化算法, 不需要“链杆”或复杂连接, 也不依赖块之 间摩擦，在垂直于单一平面方向上实现快速折叠 还原三维网格模型. 本文定义的 ETM 和 VTM 与上 述文献的空间铰链意义相近, 通过展开折叠, 支持

\section{三角网格模型的可展性.}

本文的折痕设计方法首先对每个三角面片单 独进行局部参数化, 将其映射到二维参数化平面 的原点处; 然后进行旋转和平移变换, 实现独立 且互不相连的三角面片平面布局; 最后在邻近三 角面片之间插人 ETM 和 VTM; 最终折叠还原并 逼近三维网格模型. 本文方法的处理流程如图 1 所示.

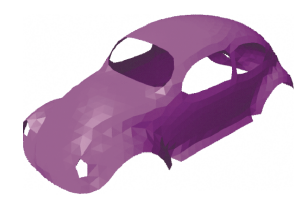

a. $M_{3 \mathrm{D}}$

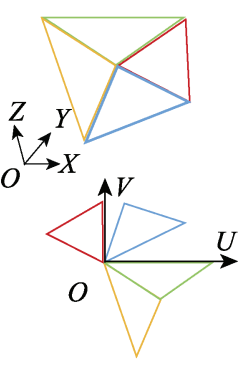

b. $M_{2 \mathrm{D}}$

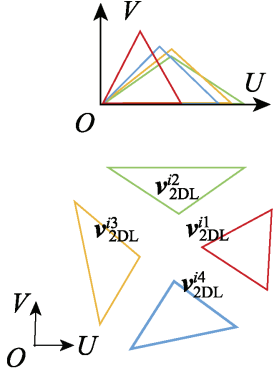

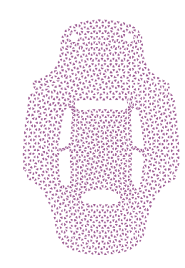

c. $M_{2 \mathrm{DL}}$

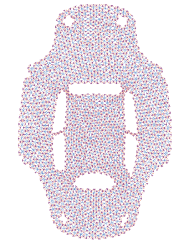

d. $M_{\mathrm{TM}}$

图 1 本文方法流程

\section{2 本文方法}

对于三维空间的网格模型 $M_{3 \mathrm{D}}\left(F_{3 \mathrm{D}}, E_{3 \mathrm{D}}, V_{3 \mathrm{D}}\right)$, 其中, $F_{3 \mathrm{D}}, E_{3 \mathrm{D}}$ 和 $V_{3 \mathrm{D}}$ 分别表示模型 $M_{3 \mathrm{D}}$ 的三角面 片集合、边集合和顶点集合，并记 $H_{3 \mathrm{D}}\left(\boldsymbol{h}_{3 \mathrm{D}}^{1}, \boldsymbol{h}_{3 \mathrm{D}}^{2}, \cdots\right.$, $\boldsymbol{h}_{3 \mathrm{D}}^{3 m}$ ) 为模型 $M_{3 \mathrm{D}}$ 的半边集合.

对于输人三维网格模型 $M_{3 \mathrm{D}}$, 设 $M_{2 \mathrm{D}}\left(F_{2 \mathrm{D}}\right.$, $\left.E_{2 \mathrm{D}}, V_{2 \mathrm{D}}\right)$ 为 $M_{3 \mathrm{D}}$ 的局部刚性参数化模型 ${ }^{[1]}$, 并记 为 $f_{1}: M_{3 \mathrm{D}} \rightarrow M_{2 \mathrm{D}}$. 对于局部参数之后的模型 $M_{2 \mathrm{D}}\left(F_{2 \mathrm{D}}, E_{2 \mathrm{D}}, V_{2 \mathrm{D}}\right)$, 其平面布局分为旋转变换和 平移变换 2 步. 记 $M_{2 \mathrm{D}}\left(F_{2 \mathrm{D}}, E_{2 \mathrm{D}}, V_{2 \mathrm{D}}\right)$ 的最终平面 布局模型为 $M_{2 \mathrm{DL}}\left(F_{2 \mathrm{DL}}, E_{2 \mathrm{DL}}, V_{2 \mathrm{DL}}\right)$, 本节将构造 平面布局映射 $f_{2}: M_{2 \mathrm{D}} \rightarrow M_{2 \mathrm{DL}}$. 对于参数空间坐 标系 $O U V$ 内的局部参数化模型 $M_{2 \mathrm{D}}$, 其三角面片 集合记为 $F_{2 \mathrm{D}}=\left\{\boldsymbol{f}_{2 \mathrm{D}}^{t}\right\}$. 本节首先构造旋转变换矩阵 $\boldsymbol{R}$ 使 $M_{2 \mathrm{DR}}=\boldsymbol{R} \cdot M_{2 \mathrm{D}}$, 然后构造平移变换矩阵 $\boldsymbol{T}$ 使 $M_{2 \mathrm{DL}}=\boldsymbol{T}+M_{2 \mathrm{DR}}$, 并记旋转平移后的模型 $M_{2 \mathrm{DL}}$ 为 最终平面布局模型, 则仿射映射 $f_{2}: M_{2 \mathrm{D}} \rightarrow M_{2 \mathrm{DL}}$ 重写为 $f_{2}: M_{2 \mathrm{D}} \rightarrow M_{2 \mathrm{DR}} \rightarrow M_{2 \mathrm{DL}}$.

在得到三维网格模型 $M_{3 \mathrm{D}}$ 的平面布局 $M_{2 \mathrm{DL}}$ 之后，在 $M_{3 \mathrm{D}}$ 上原本相邻三角面片在 $M_{2 \mathrm{DL}}$ 上被离 散分布在平面参数空间内, 由此导致相邻三角面 片之间存在缝隙, 在此基础上设计 ETM 和 VTM.

\section{1 局部参数化}

对于三维网格模型 $M_{3 \mathrm{D}}$ 上三角面片 $\boldsymbol{f}_{3 \mathrm{D}}^{t}\left(\boldsymbol{v}_{t}^{0}\right.$, $\left.\boldsymbol{v}_{t}^{1}, \boldsymbol{v}_{t}^{2}\right), t$ 表示三角面片序号; $\boldsymbol{v}_{t}^{0}, \boldsymbol{v}_{t}^{1}, \boldsymbol{v}_{t}^{2}$ 为该三角 面片的 3 个顶点, 在局部刚性参数化之后, 顶点 $v_{t}^{0}$ 位于参数坐标系 $O U V$ 的原点 $O$ 处, 顶点 $v_{t}^{0}$ 与顶点 $\boldsymbol{v}_{t}^{1}$ 所在的半边 $\boldsymbol{~}_{2 \mathrm{D}}^{i}$ 长度保持不变, 且在参数空间坐 标系 $O U V$ 中与 $O U$ 轴重合, 以此确定第 3 个顶点 的位置, 从而得到局部参数化后的三角面片 $\boldsymbol{f}_{2 \mathrm{D}}^{t}\left(\boldsymbol{v}_{t}^{0}, \boldsymbol{v}_{t}^{1}, \boldsymbol{v}_{t}^{2}\right)$.

图 2a 所示为 $M_{3 \mathrm{D}}$ 上顶点 $\boldsymbol{v}_{3 \mathrm{D}}^{i}$ 的 1-ring 邻域内 的三角面片集合; 图 $2 \mathrm{~b}$ 所示为该三角面片集合在 局部参数化后在参数化坐标系下的分布情况.

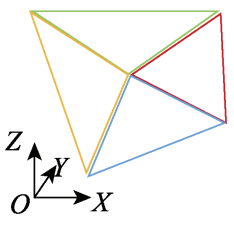

a. 输人 $M_{3 \mathrm{D}}$

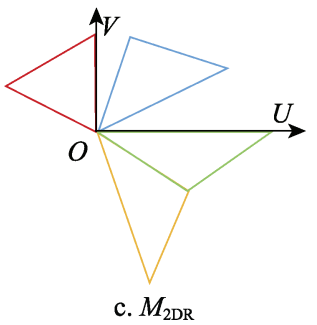

图 2 局部参数化及平面布局

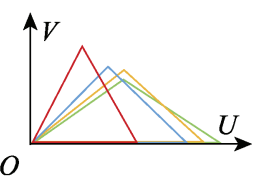

b. $M_{2 \mathrm{D}}$

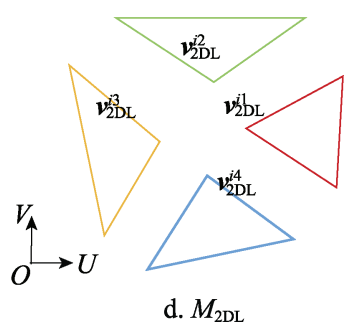

d. $M_{2 \mathrm{DL}}$

\section{2 旋转变换}

对于局部参数化模型 $M_{2 \mathrm{D}}$, 记其旋转后的模 
型为 $M_{2 \mathrm{DR}}$, 它们之间的映射关系如图 $2 \mathrm{~b}$ 与图 $2 \mathrm{c}$ 之间的旋转变换所示, 对应的旋转变换矩阵 $\boldsymbol{R}$ 构 造方法如下.

对于 $M_{2 \mathrm{D}}$ 上三角面片 $\boldsymbol{f}_{2 \mathrm{D}}^{t} \in F_{2 \mathrm{D}}$, 记 $\boldsymbol{f}_{2 \mathrm{D}}^{t}$ 位于 原点的 2 个顶点参数坐标分别为 $v_{2 \mathrm{D}}^{i}$ 和 $v_{2 \mathrm{D}}^{j}$, 并记 它们绕原点作旋转变换后得到的 2 个新的参数坐 标为 $\boldsymbol{v}_{2 \mathrm{DR}}^{i}$ 和 $\boldsymbol{v}_{2 \mathrm{DR}}^{j}$, 设 $N_{2 \mathrm{D}}^{i}$ 和 $N_{2 \mathrm{DR}}^{i}$ 分别为顶点 $\boldsymbol{v}_{2 \mathrm{D}}^{i}$ 和 $\boldsymbol{v}_{2 \mathrm{DR}}^{i}$ 的 1-ring 邻域区域, 则存在旋转矩阵 $\boldsymbol{R}_{i}{ }^{[3]}$ 使

$$
\left(\boldsymbol{v}_{2 \mathrm{DR}}^{i}-\boldsymbol{v}_{2 \mathrm{DR}}^{j}\right)=\boldsymbol{R}_{i}\left(\boldsymbol{v}_{2 \mathrm{D}}^{i}-\boldsymbol{v}_{2 \mathrm{D}}^{j}\right), j \in N_{2 \mathrm{D}}^{i}
$$

记协方差矩阵 $\boldsymbol{S}_{i}=\sum_{j \in N_{2 \mathrm{D}}^{i}} w_{i j} \boldsymbol{e}_{2 \mathrm{D}}^{i j}\left(\boldsymbol{e}_{2 \mathrm{DR}}^{i j}\right)^{\mathrm{T}}$, 其中, $w_{i j}$ 为 $\boldsymbol{f}_{2 \mathrm{D}}^{t}$ 内夹角余切值; $e_{2 \mathrm{D}}^{i j}$ 为顶点 $v_{2 \mathrm{D}}^{i}$ 和顶点 $v_{2 \mathrm{D}}^{j}$ 之 间的边长. 对协方差矩阵进行奇异值分解 $\boldsymbol{S}_{i} \rightarrow \boldsymbol{U} \Sigma \boldsymbol{V}^{\mathrm{T}}$, 则有旋转变换矩阵 $\boldsymbol{R}_{i}=\boldsymbol{U} \boldsymbol{V}^{\mathrm{T}}$. 最小 化能量函数为

$$
E_{\mathrm{R}}\left(N_{2 \mathrm{D}}^{i}, N_{2 \mathrm{DR}}^{i}\right)=\sum_{j \in N_{2 \mathrm{D}}^{i}} w_{i j}\left\|\left(\boldsymbol{v}_{2 \mathrm{DR}}^{i}-\boldsymbol{v}_{2 \mathrm{DR}}^{j}\right)-\boldsymbol{R}_{i}\left(\boldsymbol{v}_{2 \mathrm{D}}^{i}-\boldsymbol{v}_{2 \mathrm{D}}^{j}\right)\right\|^{2} .
$$

可得最优旋转变换矩阵 $\boldsymbol{R}_{i}$ 和旋转后的参数化坐标 $\boldsymbol{v}_{2 \mathrm{DR}}^{i}$ 和 $\boldsymbol{v}_{2 \mathrm{DR}}^{j}$.

\section{3 平移变换}

对于旋转变换后的模型 $M_{2 \mathrm{DR}}$, 记其平移后的 模型为 $M_{2 \mathrm{DL}}$, 它们之间的映射如图 $2 \mathrm{c}$ 与图 $2 \mathrm{~d}$ 之 间的平移变换所示, 对应的平移变换矩阵 $\boldsymbol{T}$ 构造 方法如下。

对于旋转变换后的模型 $M_{2 \mathrm{DR}}$ 上的顶点参数 坐标 $\boldsymbol{v}_{2 \mathrm{DR}}^{i}$ 和 $\boldsymbol{v}_{2 \mathrm{DR}}^{j}$ 及它们所在三角面片 $\boldsymbol{f}_{2 \mathrm{DR}}^{t}$, 在平 移过程中, 将 $\boldsymbol{f}_{2 \mathrm{DR}}^{t}$ 按照其 $n$-rings 邻域进行平移. 对于模型 $M_{2 \mathrm{DR}}$ 顶点 $\boldsymbol{v}_{2 \mathrm{DR}}^{i}$, 其平移变换过程为

$$
\boldsymbol{v}_{2 \mathrm{DL}}^{i}=\boldsymbol{T}_{i}^{+} b+\left(\boldsymbol{I}_{\mathrm{Tarns}}+\boldsymbol{T}_{i}\right) \boldsymbol{v}_{2 \mathrm{DR}}^{i}
$$

其中, $\boldsymbol{v}_{2 \mathrm{DL}}^{i}$ 为平移后的参数坐标; $\boldsymbol{T}_{i}^{+}$为 $\boldsymbol{T}_{i}$ 的伪逆 矩阵, $\boldsymbol{T}_{i}^{+}=\boldsymbol{T}_{i}^{\mathrm{T}}\left(\boldsymbol{T}_{i} \boldsymbol{T}_{i}^{\mathrm{T}}\right)^{-1} ; b$ 为任意计算参数. 构建 能量函数 $E_{\mathrm{L}}$, 使其最小化可得最优平移变换矩阵 $T_{i}$ 和旋转后的顶点坐标 $v_{2 \mathrm{DL}}^{i}$ 和 $v_{2 \mathrm{DL}}^{j}$, 即

$$
\begin{aligned}
& E_{\mathrm{L}}\left(N_{2 \mathrm{DR}}^{i}, N_{2 \mathrm{DL}}^{i}\right)= \\
& \quad \sum_{j \in N_{2 \mathrm{DR}}^{i}} c_{0} \cdot\left\|c_{1}\left(\boldsymbol{v}_{2 \mathrm{DL}}^{i}-\boldsymbol{v}_{2 \mathrm{DL}}^{j}\right)-c_{2} \boldsymbol{T}_{i}\left(\boldsymbol{v}_{2 \mathrm{DR}}^{i}-\boldsymbol{v}_{2 \mathrm{DR}}^{j}\right)\right\|^{2}(3)
\end{aligned}
$$

其中, $c_{0}: c_{1}: c_{2}=e_{\mathrm{ave}}^{2}: 1: 1$ 以限制模型伸缩, $e_{\mathrm{Ave}}$ 为 $M_{3 \mathrm{D}}$ 的平均边长. 为得到局部最优 $\boldsymbol{f}_{2 \mathrm{DL}}^{t}$, 以线搜 索对其独立计算平移参数 $\lambda$, 则有

$$
\boldsymbol{v}_{2 \mathrm{DL}}^{i}-\boldsymbol{v}_{2 \mathrm{DL}}^{j}=\left(\boldsymbol{v}_{2 \mathrm{DR}}^{i}-\boldsymbol{v}_{2 \mathrm{DR}}^{j}\right)-\lambda \nabla E_{\mathrm{L}}^{k}
$$

其中, $\lambda \geqslant 0 ; \nabla E_{\mathrm{L}}^{k}$ 为 $E_{\mathrm{L}}$ 的梯度线搜索方向; $k$ 为 迭代次数. 记 $\varphi(\lambda)=\left(v_{2 \mathrm{DR}}^{i}-v_{2 \mathrm{DR}}^{j}\right)-\lambda \nabla E_{\mathrm{L}}^{k}$, 从而将 求局部最优 $\boldsymbol{f}_{2 \mathrm{DL}}^{t}$ 问题转换为求 $\varphi(\lambda)$ 的极小点问题, 当 $\varphi^{\prime}(\lambda)=\nabla f\left(\left(v_{2 \mathrm{DR}}^{i}-v_{2 \mathrm{DR}}^{j}\right)^{k}-\lambda \nabla E_{\mathrm{L}}^{k}\right) \nabla E_{\mathrm{L}}^{k}=0$ 时, 可 求得使 $\varphi(\lambda)$ 最小的 $\lambda$ 值. 如图 3 所示, $\boldsymbol{f}_{2 \mathrm{DL}}^{t}$ 在二维 参数空间平面得到分布最均匀.

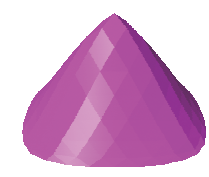

a. $M_{3 \mathrm{D}}$

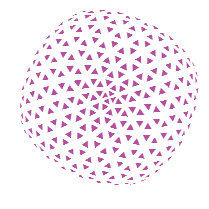

b. $M_{2 \mathrm{DL}}$
图 3 Gaussian 模型平面布局结果

\subsection{ETM 设计}

如图 3 所示, 在得到三维网格模型 $M_{3 \mathrm{D}}$ 的平 面布局 $M_{2 \mathrm{DL}}$ 之后， $M_{3 \mathrm{D}}$ 上原本相邻的三角面片在 $M_{2 \mathrm{DL}}$ 上被离散分布在平面参数空间内, 由此导致 $M_{3 \mathrm{D}}$ 上原本相邻的三角面片的公用边 $\boldsymbol{e}_{3 \mathrm{D}}^{n}$ 在 $M_{2 \mathrm{DL}}$ 上被分解为 2 条半边, 分别记为 $\boldsymbol{h}_{2 \mathrm{D}}^{n 1}$ 和 $\boldsymbol{h}_{2 \mathrm{D}}^{n 2}$; 原本 相邻的三角面片的公用顶点 $v_{3 \mathrm{D}}^{i}$ 在 $M_{2 \mathrm{DL}}$ 上被分解 为 $k$ 个顶点, 分别记为 $v_{2 \mathrm{DL}}^{i 1}, v_{2 \mathrm{DL}}^{i 2}, \cdots, v_{2 \mathrm{DL}}^{i k}$.

如图 4 所示, 本节参照文献[2]方法, 在 2 条半 边 $\boldsymbol{h}_{2 \mathrm{D}}^{n 1}$ 和 $\boldsymbol{h}_{2 \mathrm{D}}^{n 2}$ 围成的四边形区域内插人 ETM 设计, 并在 $k$ 个顶点 $v_{2 \mathrm{DL}}^{i 1}, v_{2 \mathrm{DL}}^{i 2}, \cdots, v_{2 \mathrm{DL}}^{i k}$ 围成的 $k$ 边形内 插人 VTM 设计.

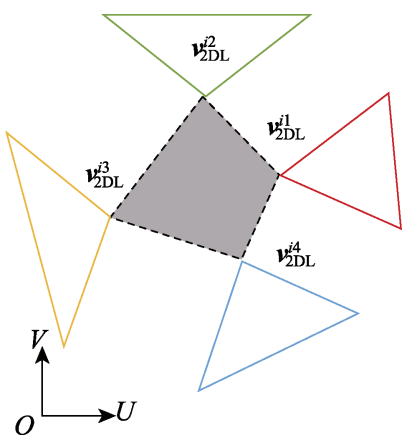

图 4 折痕区域

对于 $M_{2 \mathrm{DL}}$ 空间内的半边 $\boldsymbol{h}_{2 \mathrm{D}}^{n 1}$, 若其不存在对 边, 则半边 $\boldsymbol{h}_{2 \mathrm{D}}^{n 1}$ 为边界边, 不做处理; 若其存在对 边 $\boldsymbol{h}_{2 \mathrm{D}}^{n 2}$, 则需要构造 ETM. 如图 5a 所示, 对于 $\mathrm{M}_{3 \mathrm{D}}$ 的 2 个相邻三角面片, 其公用边 $\boldsymbol{e}_{3 \mathrm{D}}^{n}$ 在 $M_{2 \mathrm{DL}}$ 空间内分解为 2 条半边 $\boldsymbol{h}_{2 \mathrm{D}}^{n 1}$ 和 $\boldsymbol{h}_{2 \mathrm{D}}^{n 2}$ 后, 其端点分别 记为起点 $A$ 、终点 $B$, 以及起点 $B^{\prime}$ 、终点 $A^{\prime}$, 按 照顺时针排序得到四边形 $A B B^{\prime} A^{\prime}$, 由此产生的四 
边形 ETM 区域有 2 种情况，一种是图 $5 \mathrm{~b}$ 所示的不 规则四边形区域; 另一种是图 5c 所示的 $M_{2 \mathrm{DL}}$ 空间 内的等腰梯形区域.

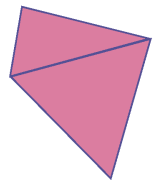

a. $M_{3 \mathrm{D}}$

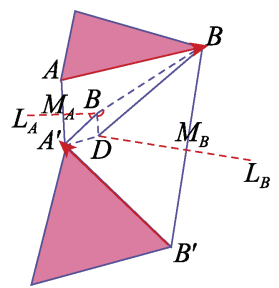

b. 不规则形式

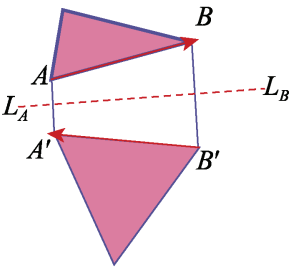

c. 对称形式
图 52 种 $\mathrm{ETM}$

对于图 $5 \mathrm{~b}$ 中的不规则四边形区域, 直线 $L_{A}$ 和 $L_{B}$ 分别为 $A A^{\prime}$ 和 $B B^{\prime}$ 的中垂线, $M_{A}$ 和 $M_{B}$ 分别为 $A A^{\prime}$ 和 $B B^{\prime}$ 的中点, 则 $L_{B}$ 上存在点 $D$, 满足 $\angle B M_{A} D=\pi / 2$, 且 $L_{A}$ 上存在点 $C$, 满足 $\angle M_{A} C A^{\prime}+\angle D C B=\pi$, 以保证折叠后构成同一条 $\boldsymbol{e}_{3 \mathrm{D}}^{n}$, 连接 $A^{\prime} C, B D$ 作为脊折痕, $M_{A} C, M_{B} D$ 作为 谷折痕. 对于图 $5 \mathrm{c}$ 中对称形式的等腰梯形区域, 中垂线 $L_{A}$ 与 $L_{B}$ 重合, 即为脊折痕线, 沿折痕线对 称折叠即可. ETM 设计如图 6 所示.

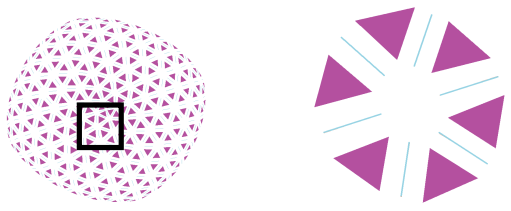

图 $6 \mathrm{ETM}$ 对称形式

\subsection{VTM 设计}

如图 7a 所示, 对于 $M_{3 \mathrm{D}}$ 的顶点 $v_{3 \mathrm{D}}^{i}$, 设其在 $M_{2 \mathrm{DL}}$ 上的映射为 $\boldsymbol{v}_{2 \mathrm{DL}}^{i 1}, v_{2 \mathrm{DL}}^{i 2}, \cdots, v_{2 \mathrm{DL}}^{i k}$, 则构成具有 $k$ 个顶点的 VTM 多边形如图 7b 所示, 记为 $\mathrm{VTM}^{i}$. 基于所有 $\mathrm{VTM}^{i}$ 多边形的顶点, 以增量法构造 Voronoi 图如图 7c 所示, 记所得 Voronoi 图的全部 站点为 $\boldsymbol{v}_{\mathrm{Vor}}^{0}, \boldsymbol{v}_{\mathrm{Vor}}^{1}, \boldsymbol{v}_{\mathrm{Vor}}^{2} \cdots$. 显然, 所得 Voronoi 图落在 ETM 多边形内部的站点属于无效站点, 只有落在 $\mathrm{VTM}^{i}$ 多边形内部部分站点可以用于构造顶点折 叠铰链的折痕. 本文排除无效站点的约束条件为

$$
\left(\left(\boldsymbol{v}_{\mathrm{Vor}}^{n}-\boldsymbol{v}_{2 \mathrm{DL}}^{i}\right) \times \boldsymbol{e}_{2 \mathrm{DL}}^{i, i+1}\right) \times\left(\left(\boldsymbol{v}_{\mathrm{Vor}}^{n}-\boldsymbol{v}_{2 \mathrm{DL}}^{i+1}\right) \times \boldsymbol{e}_{2 \mathrm{DL}}^{i+1, i+2}\right)>0 .
$$

如图 7d 所示, 对于有效站点, 分别连接与其 最近的 $\mathrm{VTM}^{i}$ 多边形顶点, 即为 VTM, 从而完成 VTM 的构造. 图 8 所示为 Gaussian 模型的局部 VTM 实例, 其中增量法构造 Voronoi 图的详细过程 如图 9 所示.

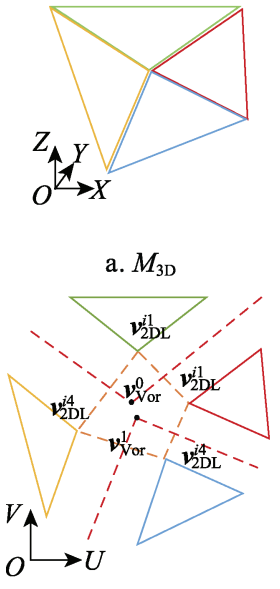

c. Voronoi 图

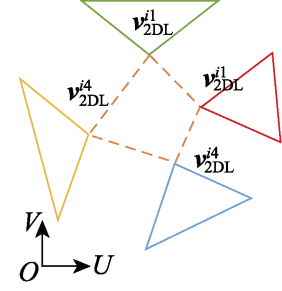

b. VTM

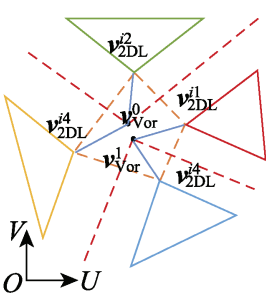

d. 构建折痕
图 7 VTM 构造示意图

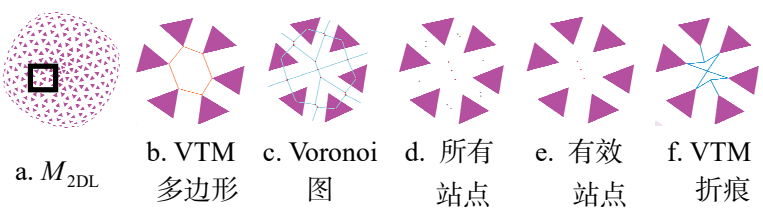

图 8 Gaussian 模型局部 VTM 设计

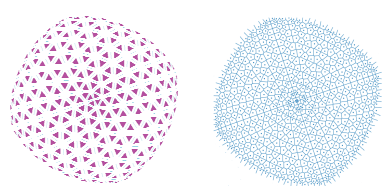

$\begin{array}{lll}\text { a. } M_{2 \mathrm{DL}} & \text { b. Voronoi 图 c. Voronoi 站点 }\end{array}$
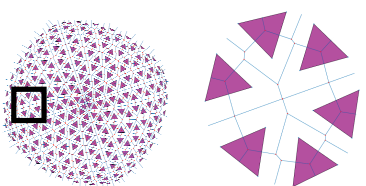

d. 局部放大
图 9 增量法构造 Voronoi 图

\section{3 本文方法流程}

本文方法主要包括平面布局和空间折痕设计 2 个步骤. 其中平面布局又包括局部参数化、旋转 变换、平移变换 3 个部分; 空间折痕设计又包括 ETM 设计和 VTM 设计. 下面将按照此顺序介绍本 文方法的流程.

(1) 局部参数化. 对于输人的三维网格模型 $M_{3 \mathrm{D}}$ ，将 $\boldsymbol{f}_{3 \mathrm{D}}^{t}\left(\boldsymbol{v}_{t}^{0}, \boldsymbol{v}_{t}^{1}, \boldsymbol{v}_{t}^{2}\right)$ 刚性局部参数化, 其中, $\boldsymbol{v}_{t}^{0}$ 位于参数坐标系 $O U V$ 的原点 $O$ 处; $v_{t}^{0}$ 与 $v_{t}^{1}$ 所在半 边 $\boldsymbol{h}_{2 \mathrm{D}}^{i}$ 与 $O U$ 轴重合, 得到局部参数化模型 $M_{2 \mathrm{D}}$.

(2) 旋转、平移变换. 平面布局变换包括旋转 变换和平移变换. 对于局部参数化模型 $M_{2 \mathrm{D}}$, 其 中旋转变换将 $O U$ 轴上 2 点 $v_{2 \mathrm{D}}^{i}$ 和 $v_{2 \mathrm{D}}^{j}$ 经绕原点的 旋转变换得到新的参数坐标 $v_{2 \mathrm{DR}}^{i}$ 和 $v_{2 \mathrm{DR}}^{j}$, 记旋转 
变换后的模型为 $M_{2 \mathrm{DR}}$; 然后将 $M_{2 \mathrm{DR}}$ 中的 $\boldsymbol{f}_{2 \mathrm{DR}}^{t}$ 按 照其 $n$-rings 邻域进行平移变换, $\boldsymbol{v}_{2 \mathrm{DR}}^{i}$ 经平移变换 后得到的点记为 $\boldsymbol{v}_{2 \mathrm{DL}}^{i}$, 并引人平移参数 $\lambda$ 控制 $\boldsymbol{f}_{2 \mathrm{DL}}^{t}$ 平面结果, 记平移变换后的模型为 $M_{2 \mathrm{DL}}$. 最 终, 记平面布局变换后得到的最终平面布局模型 为 $M_{2 \mathrm{DL}}$. 布局算法详细步骤如下所示.

输人. $M_{2 \mathrm{D}}$ 中各点 $\boldsymbol{v}_{2 \mathrm{D}}^{i}$.

输出. $M_{2 \mathrm{DL}}$ 中各点 $\boldsymbol{v}_{2 \mathrm{DL}}^{i}$.

Step1. 计算协方差矩阵 $\boldsymbol{S}_{i}=\sum_{j \in N_{2 \mathrm{D}}^{N}} w_{i j} \boldsymbol{e}_{2 \mathrm{D}}^{i j}\left(\boldsymbol{e}_{2 \mathrm{DR}}^{i j}\right)^{\mathrm{T}}$.

Step2. 对 $\boldsymbol{S}_{i}$ 进行奇异值分解, 得旋转变换矩阵 $\boldsymbol{R}_{i}$.

Step3. 基于式(1)将构成 $\boldsymbol{e}_{2 \mathrm{D}}^{i j}$ 的点 $\boldsymbol{v}_{2 \mathrm{D}}^{i}$ 和 $\boldsymbol{v}_{2 \mathrm{D}}^{j}$ 绕原点 坐标旋转变换, 得到点 $v_{2 \mathrm{DR}}^{i}$ 和 $v_{2 \mathrm{DR}}^{j}$.

Step4. 根据新点 $\boldsymbol{v}_{2 \mathrm{DR}}^{i}$ 和 $\boldsymbol{v}_{2 \mathrm{DR}}^{j}$, 基于式(3)(4)构建平 移变换矩阵 $\boldsymbol{T}$.

Step5. 将 $\boldsymbol{f}_{2 \mathrm{DR}}^{t}$ 按照其 $n$-rings 邻域进行平移, 基于 式(2), $\boldsymbol{v}_{2 \mathrm{DR}}^{j}$ 平移变换得到 $\boldsymbol{v}_{2 \mathrm{DL}}^{j}$.

Step6. 更新布局后的点为 $\boldsymbol{v}_{2 \mathrm{DL}}^{i}$.

(3) 折痕设计. 设计 ETM 和 VTM, 并将其插 人 $M_{2 \mathrm{DL}}$ 缝隙. 首先, 根据 $M_{2 \mathrm{DL}}$ 中 $\boldsymbol{f}_{2 \mathrm{DL}}^{t}$ 分布是否 均匀、 ETM 是否为对称图形, 设计 2 种 EMT; 其 次, 因为 VTM 均为不规则多边形, 以增量法构造 $\mathrm{VTM}^{i}$ 顶点集合的 Voronoi 图，基于约束构造有效 站点, 分别连接与其最近的 VTM ${ }^{i}$ 顶点, 得到 VTM 折痕；最终完成折痕模型 $M_{\mathrm{TM}}$ 构造.

\section{4 实验结果与分析}

本文实验环境为 Intel(R) Core(TM) i7 处理器, 主频 $3.4 \mathrm{GHz}$, 内存 $32 \mathrm{~GB}$, 操作系统为 64 位 Windows. 编程语言环境为 Microsoft Visual Studio $2010 \mathrm{C}++$.

\section{1 折痕设计效果}

图 $10 \mathrm{~b}$ 为对应三角网格模型平面布局后的 ETM 和 VTM 设计效果. 表 1 给出了相关的实验数 据, 如方法运行时间 $T$ (包含预处理阶段)、模型顶 点数 $N_{\mathrm{Ver}}$ (与 $N_{\mathrm{VTM}}$ 数相同)、三角面片数 $N_{\mathrm{Tri}}$ 、边

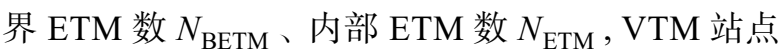
数 $V_{\mathrm{V} \text { or }}$ 、不稳定性 $I$ 等. 不稳定性的计算公式为 $I=3 v-e-6=e_{0}-3, v, e$ 和 $e_{0}$ 分别为 $M_{2 \mathrm{D}}$ 的顶点 数、总边数和边界上的边数. 实验结果表明, 文献 [2]方法仅可应用在多边形数目少于 400 的模型, 而本文方法在处理多边形数目为 256 1 759 的模型 均可得到均匀的平面布局效果; 本文方法运行时
间与模型规模成正比，在本文实验模型中，运行时 间均不超过 $0.45 \mathrm{~s}$ ，且最快为 $0.077 \mathrm{~s}$.

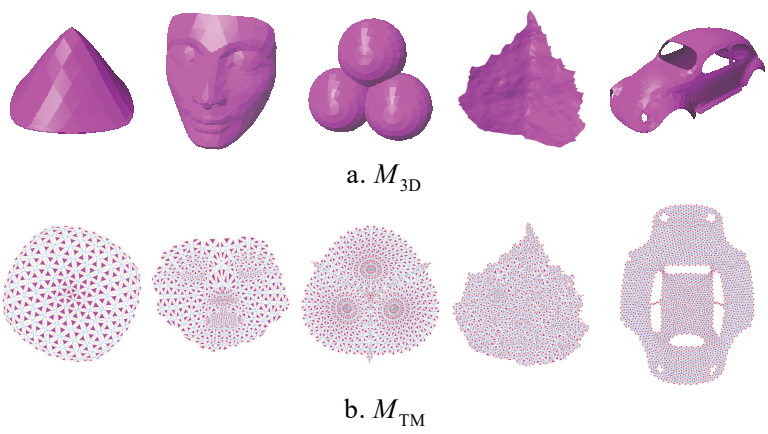

图 10 部分模型的 ETM 和 VTM 设计

表 1 部分实验数据

\begin{tabular}{lcccccccc}
\hline \multicolumn{1}{c}{ 模型 } & $T / \mathrm{s}$ & $N_{\mathrm{Ver}}$ & $N_{\mathrm{VTM}}$ & $N_{\text {Tri }}$ & $N_{\text {BETM }}$ & $N_{\text {ETM }}$ & $V_{\text {Vor }}$ & $I$ \\
\hline Gaussian & 0.077 & 145 & 145 & 256 & 32 & 368 & 182 & 29 \\
Face & 0.125 & 299 & 299 & 562 & 34 & 826 & 224 & 31 \\
Ball & 0.211 & 547 & 547 & 1032 & 60 & 1518 & 453 & 57 \\
Leaf & 0.227 & 664 & 664 & 1181 & 145 & 1699 & 371 & 142 \\
Car & 0.449 & 984 & 984 & 1759 & 227 & 2525 & 530 & 224 \\
\hline
\end{tabular}

本文方法对于亏格为 10 的 Car 模型依然能够 得到较好的平面布局效果, 而 MEAN 方法对于 $\mathrm{Car}$ 模型无法得到其平面布局结果，表明本文方法对亏 格不为 0 的三维网格模型仍具有健壮性. 本文平面 布局、ETM 和 VTM 设计的更多效果如图 11 所示.

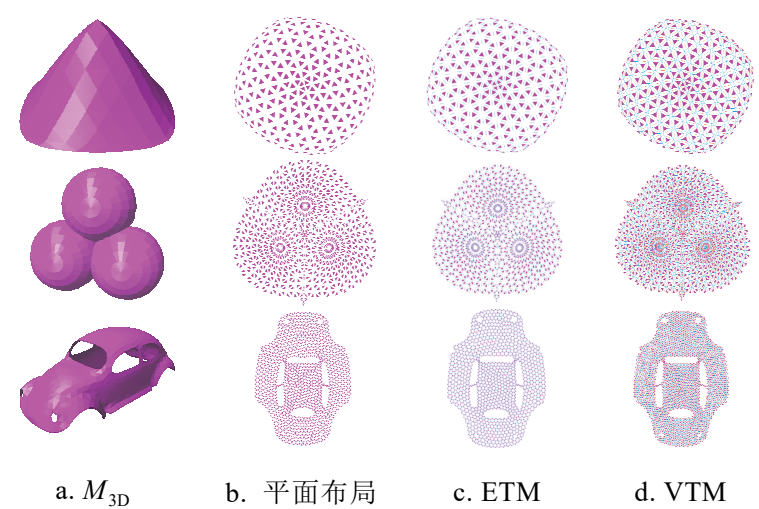

图 11 各模型设计过程效果图

\section{2 平面布局误差分析}

本文方法以局部参数化做预处理, 零误差实 现平面布局过程中 $M_{2 \mathrm{D}}, M_{2 \mathrm{DL}}$ 与 $M_{3 \mathrm{D}}$ 之间的转换. 首先, 对 $M_{3 \mathrm{D}}$ 刚性局部参数化, 三角形保持原有 形状, 零误差转换为 $M_{2 \mathrm{D}}$; 其次, 对 $M_{2 \mathrm{D}}$ 进行 ARAP 刚性旋转变换, 与 ASAP(as similar as pos- 
sible)相比, 三角面片依然保持原有形状, 零误差 转换为 $M_{2 \mathrm{DR}}$, 而 ASAP 方法为了保证三角面片之 间的连通性，选择以局部改变三角面片形状的方 法得到局部参数化模型, 因此 ASAP方法不能够保 证模型刚性, 最终结果仍存在一定误差; 最后, 对 $M_{2 \mathrm{DR}}$ 进行平移变换, 仍保证零误差转换为 $M_{2 \mathrm{DL}}$, 即平面布局结果.

(1) 亏格. 比较而言, LSCM 为保角映射参数 化, 通过参数化局部保留质心坐标, 达到误差最小 的目的 ${ }^{[13]}$, 但对于存在非边界奇异点的模型, 该方 法无法准确找到边界(如 Gaussian 模型). MEAN 方 法可固定边界形状 ${ }^{[14]}$, 边界上每相邻 2 点之间的距 离是相等的, 但仅可对单边界模型进行参数化, 即 仅适用于亏格为 0 的单边界模型, 无法处理有亏格 的模型(如 Car 模型亏格为 10), 且该方法误差较大.
(2) 失真度. 本文 ARAP 局部参数化与 LSCM 方法和 MEAN 方法的失真度对比结果如表 2 所示. 对于参数化模型 $M_{\text {Para }}$, 其面积失真度记为 $D_{\text {Area }}=$ $\sum A_{3 \mathrm{D}} / \sum A_{\text {para }}$, 其中, $A_{3 \mathrm{D}}$ 为 $M_{3 \mathrm{D}}$ 面积总和, $A_{\text {Para }}$ 为 $M_{\text {Para }}$ 面积总和; 其角度失真度记为 $D_{\text {Angle }}=\sum_{i=0}\left(\sum R_{3 \mathrm{D}}^{i} / \sum R_{\text {Para }}^{i}\right) / 3$, 其中, $R_{3 \mathrm{D}}^{i}$ 为 $M_{3 \mathrm{D}}$ 每个 $\boldsymbol{f}_{3 \mathrm{D}}^{t}$ 内角角度, $R_{\text {Para }}^{i}$ 为 $M_{\text {Para }}$ 每个 $\boldsymbol{f}_{\text {Para }}^{t}$ 内角角 度; 其边长失真度记为 $D_{\text {Length }}=$ $\sum_{i=0}\left(\sum L_{3 \mathrm{D}}^{i} / \sum L_{\text {Para }}^{i}\right) / 3$, 其中, $L_{3 \mathrm{D}}$ 为 $M_{3 \mathrm{D}}$ 所有 $\boldsymbol{e}_{3 \mathrm{D}}^{n}$ 总和, $L_{\text {Para }}$ 为 $M_{\text {Para }}$ 中 $\boldsymbol{e}_{\text {Para }}^{n}$ 总和. 当失真度为 1 时, 参数化模型 $M_{\text {Para }}$ 与原始模型 $M_{3 \mathrm{D}}$ 之间的形状差 异最小. 显然, ARAP 方法各项失真度值最稳定, 均趋近于 1 .

表 2 本文与 LSCM 和 MEAN 方法的各项失真度对比

\begin{tabular}{|c|c|c|c|c|c|c|c|c|c|}
\hline \multirow{2}{*}{ 模型 } & \multicolumn{3}{|c|}{$\mathrm{ARAP}^{[3]}$} & \multicolumn{3}{|c|}{$\mathrm{LSCM}^{[13]}$} & \multicolumn{3}{|c|}{$\operatorname{MEAN}^{[14]}$} \\
\hline & $D_{\text {Area }}$ & $D_{\text {Angle }}$ & $D_{\text {Length }}$ & $D_{\text {Area }}$ & $D_{\text {Angle }}$ & $D_{\text {Length }}$ & $D_{\text {Area }}$ & $D_{\text {Angle }}$ & $D_{\text {Length }}$ \\
\hline Gaussian & 1.046 & 0.988 & 0.984 & & & & 1.254 & 0.969 & 1.123 \\
\hline Face & 1.010 & 1.000 & 1.008 & 2.106 & 1.000 & 1.487 & 7.674 & 1.005 & 3.217 \\
\hline Ball & 1.073 & 0.991 & 1.049 & 3.141 & 0.995 & 1.886 & 1.017 & 0.883 & 1.130 \\
\hline Leaf & 1.000 & 1.000 & 1.000 & 1.307 & 1.000 & 1.144 & 0.001 & 0.915 & 0.030 \\
\hline Car & 1.012 & 1.021 & 1.005 & 17.573 & 1.020 & 4.236 & & & \\
\hline
\end{tabular}

图 12 对比了 ARAP 局部参数化与 LSCM 和 MEAN 方法的失真度曲线. 通过以上失真度数据 可知, ARAP 方法中各模型的面积、角度和边长失 真度值均接近 1 ; 而 LSCM 方法仅在角度失真上达 到了最优效果, 边长失真度较大, 面积失真度超过 2. 由表 2 中 LSCM 算法失真度数据可知, Car 模型 面积失真度达到极大值. MEAN 方法仅在角度失真 上效果较好, 边长失真度超过 3 , 面积失真度达到 极大值, 超过 7, 面积失真度极大. ARAP 方法的 Car 模型面积、角度以及边长失真度同样接近 1 ; LSCM 方法的面积失真度值和边长失真度值超出 预期范围; MEAN 方法无法得到 Car 模型的参数化 模型. 因此, 本文采用的 ARAP 局部参数化方法失 真度最小.

(3) 标准误差. 表 3 从面积、角度和边长等 3 个方面对比分析参数化方法的测量标准误差. 其 中, 面积标准误差定义为 $\delta_{\text {Area }}=\operatorname{sqrt}\left(\sum E_{\text {Area }}^{2} / n\right)$, 边长标准误差定义为 $\delta_{\text {Length }}=\operatorname{sqrt}\left(\sum E_{\text {Length }}^{2} / 3 n\right)$,
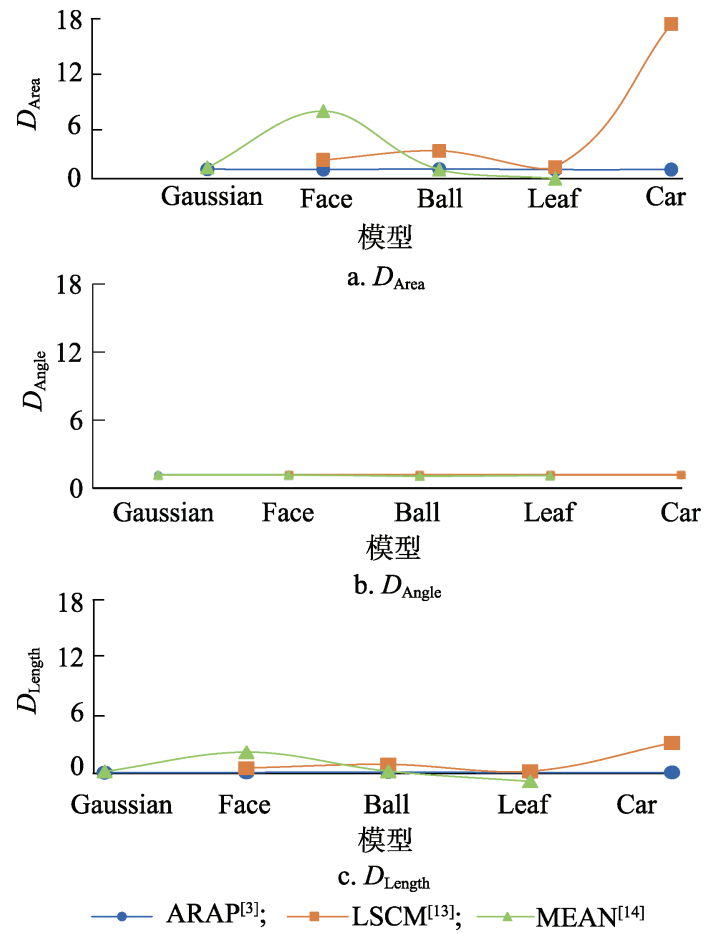

图 12 ARAP, LSCM 和 MEAN 方法的失真度曲线 
表 3 本文与 LSCM 和 MEAN 方法的标准误差对比

\begin{tabular}{|c|c|c|c|c|c|c|c|c|c|}
\hline \multirow{2}{*}{ 模型 } & \multicolumn{3}{|c|}{$\mathrm{ARAP}^{[3]}$} & \multicolumn{3}{|c|}{$\operatorname{LSCM}^{[13]}$} & \multicolumn{3}{|c|}{$\operatorname{MEAN}^{[14]}$} \\
\hline & $\delta_{\text {Area }}$ & $\delta_{\text {Angle }}$ & $\delta_{\text {Length }}$ & $\delta_{\text {Area }}$ & $\delta_{\text {Angle }}$ & $\delta_{\text {Length }}$ & $\delta_{\text {Area }}$ & $\delta_{\text {Angle }}$ & $\delta_{\text {Length }}$ \\
\hline Gaussian & 0.011 & 0.451 & 0.087 & & & & 0.052 & 1.051 & 0.592 \\
\hline Face & 0.009 & 0.022 & 0.100 & 0.531 & 4.323 & 0.011 & 0.880 & 9.094 & 0.153 \\
\hline Ball & 0.007 & 0.359 & 0.239 & 0.068 & 0.166 & 2.385 & 0.002 & 4.925 & 0.584 \\
\hline Leaf & 0.000 & 0.010 & 0.000 & 0.000 & 0.007 & 0.020 & 0.093 & 3.991 & 5.256 \\
\hline Car & 0.001 & 1.214 & 0.019 & 0.048 & 1.169 & 3.680 & & & \\
\hline
\end{tabular}

角度标准误差定义为 $\delta_{\text {Angle }}=\operatorname{sqrt}\left(\sum E_{\text {Angle }}^{2} / 3 n\right)$. 对比 ARAP, LSCM 和 MEAN 方法可知, 本文的 ARAP 方法标准误差最低.

图 13 所示为 ARAP, LSCM 以及 MEAN 方法 的面积、角度、边长标准误差曲线图. 对比数据曲 线可知: 以 ARAP 方法参数化后面积、角度、边长 标准误差值均接近于 0 , 仅有 $\mathrm{Car}$ 模型的角度标准 误差值超过 1; 以 LSCM 方法参数化后, Face 模型 的角度标准误差值超过 4, 达到极大值, Ball 模型 和 $\mathrm{Car}$ 模型边长标准误差值分别为 2.384777 和 3.680311; 以 MEAN 方法参数化, Face 模型角度标 准误差值超过 9, 达到极大值, Leaf 模型的边长、 角度标准误差值和 Ball 模型的角度标准误差值均 大于 3.5 , 超出预期范围. 因此, 在标准误差对比 中, ARAP 方法失真最小.
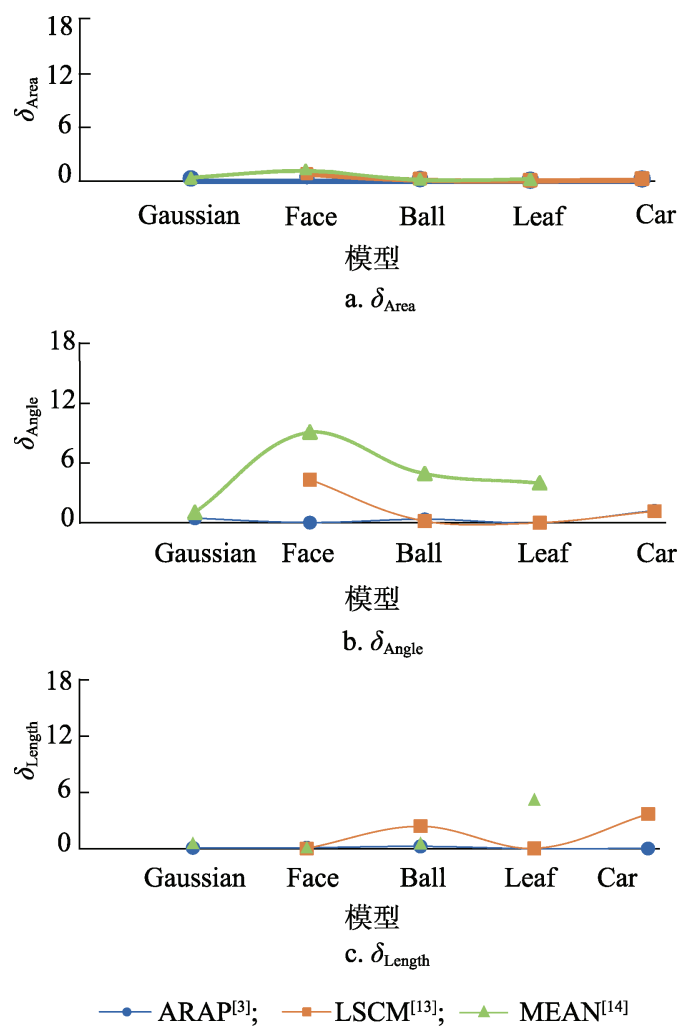

图 13 ARAP, LSCM 和 MEAN 的标准误差曲线

\section{3 平移参数 $\lambda$}

图 14 所示为本文与文献[16]方法的对比效果. 在平面布局阶段，为实现三角面片空间分布均匀化 且无碰撞, 本文以平移参数 $\lambda$ 调整 $M_{2 \mathrm{DL}}$ 布局.
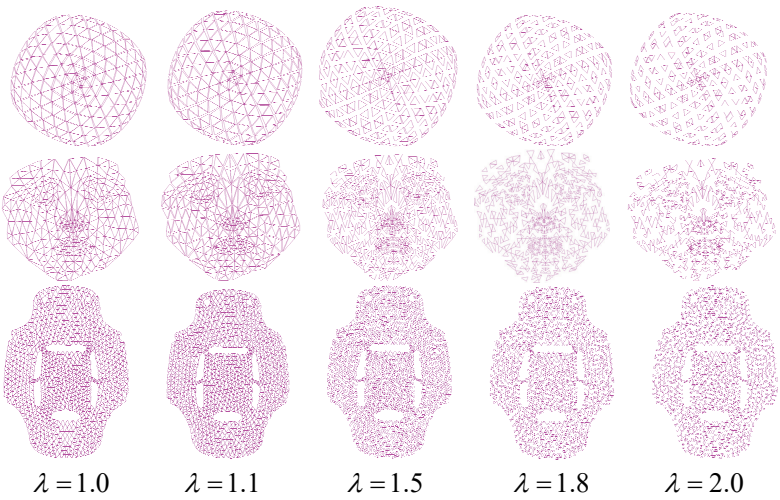

a. 质心平移 ${ }^{[16]}$
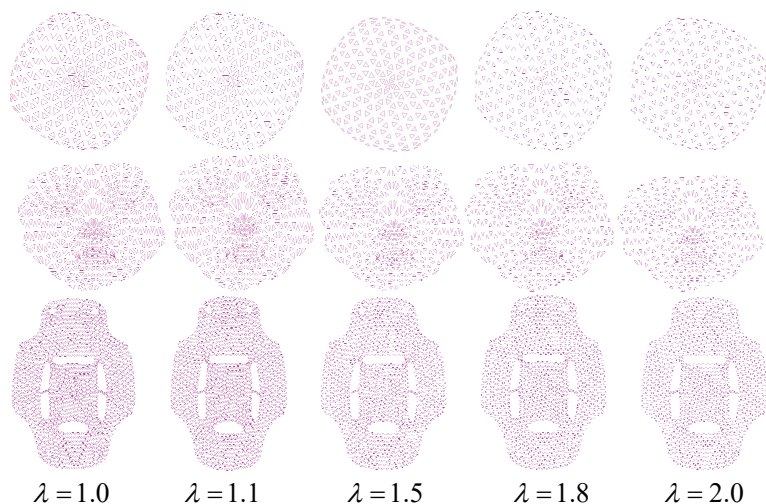

b. 本文

图 14 不同方法平移参数对平面布局的影响

文献[16]采用质心算法实现平移，记 $\boldsymbol{V}_{2 \mathrm{DL}}=$ $\boldsymbol{V}_{2 \mathrm{DR}}+\lambda\left(\boldsymbol{V}_{\mathrm{Para}}^{\mathrm{b}}-\boldsymbol{V}_{2 \mathrm{DR}}^{\mathrm{b}}\right)$, 固定 $M_{2 \mathrm{D}}$ 与 $M_{2 \mathrm{DL}}$ 三角面 片质心位置 $\boldsymbol{V}_{\mathrm{Para}}^{\mathrm{b}}, \boldsymbol{V}_{2 \mathrm{DR}}^{\mathrm{b}}$ 以确定平移方向与平移距 离, 由于未考虑相邻三角面片之间的拓扑关系, 导 致相邻三角面片产生交叉重叠; 为避免碰撞, 则必 须改变三角面片的形状.

而本文方法在旋转后直接匹配 2 组共面点集, 基于邻域信息平移, 实现了三角面片无变形、无交 叉重叠且分布较为均匀的平面布局结果. 


\section{4 平面布局的稳定性}

本文平面布局由旋转变换和平移变换 2 部分 构成, 平面布局方法的稳定性由旋转能量公式 $E_{\mathrm{R}}$ 和平移能量公式 $E_{\mathrm{L}}$ 衡量. 在计算最优平面布局变 换的迭代过程中，随着 $\left(v_{3 \mathrm{D}}^{i}-v_{3 \mathrm{D}}^{j}\right),\left(v_{2 \mathrm{DR}}^{i}-v_{2 \mathrm{DR}}^{j}\right)$ 以 及 $\left(v_{2 \mathrm{DL}}^{i}-v_{2 \mathrm{DL}}^{j}\right)$ 之间差异逐渐缩小, $M_{2 \mathrm{D}}$ 与 $M_{2 \mathrm{DR}}$ 间、 $M_{2 \mathrm{DR}}$ 与 $M_{2 \mathrm{DL}}$ 间的误差逐渐缩小, 如图 15 所 示, $E_{\mathrm{R}}$ 和 $E_{\mathrm{L}}$ 的能量曲线同时随着迭代次数的增 加逐渐减小, 最终为 0 , 表明本文平面布局方法趋 于稳定，可以得到最优 $M_{2 \mathrm{DL}}$.

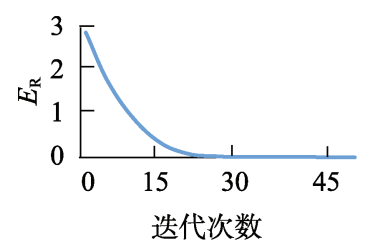

a. $E_{\mathrm{R}}$

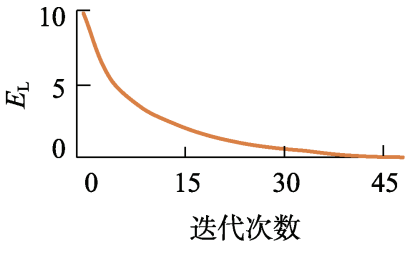

b. $E_{\mathrm{L}}$
图 15 平面布局过程中能量曲线

为验证 $M_{\mathrm{TM}}$ 折叠后与 $M_{3 \mathrm{D}}$ 保持一致, 本节给 出部分模型的边长约束能 $E_{\text {Length }}$ 、角度约束能 $E_{\mathrm{Angle}}$ 和面积约束能 $E_{\mathrm{Area}}$. 若迭代过程中, 平面布 局的约束能均趋近于 0 , 则表示迭代过程可以平面 布局的平面布局变换无误差、三角面片不变形且分 布较为均匀的情况下结束. 此时, $M_{\mathrm{TM}}$ 在折叠后 与 $M_{3 \mathrm{D}}$ 保持一致，不存在拉伸、挤压等变形.

相关约束能量 ${ }^{[4]}$ 定义如下:

边长约束能量

$$
E_{\text {Length }}=\sum_{j \in N_{2 \mathrm{D}}^{i}}\left\|\left(\boldsymbol{v}_{2 \mathrm{DL}}^{i}-\boldsymbol{v}_{2 \mathrm{DL}}^{j}\right)-P_{\mathrm{E}}\left(\boldsymbol{v}_{2 \mathrm{DL}}^{i}, \boldsymbol{v}_{2 \mathrm{DL}}^{j}\right)\right\|_{2}^{2} ;
$$

其 中, $P_{\mathrm{E}}\left(\boldsymbol{v}_{2 \mathrm{DL}}^{i}, \boldsymbol{v}_{2 \mathrm{DL}}^{j}\right)=\left\|\boldsymbol{v}_{3 \mathrm{D}}^{i}-\boldsymbol{v}_{3 \mathrm{D}}^{j}\right\| /\left\|\boldsymbol{v}_{2 \mathrm{DL}}^{i}-\boldsymbol{v}_{2 \mathrm{DL}}^{j}\right\| \times$ $\left(v_{2 \mathrm{DL}}^{i}-v_{2 \mathrm{DL}}^{j}\right)$.

角度约束能量

$$
E_{\text {Angle }}=\sum_{i \in \mathrm{AN}}\left\|\boldsymbol{R}_{2 \mathrm{DL}}^{i}-P_{\mathrm{AN}}\left(\boldsymbol{R}_{2 \mathrm{DL}}^{i}\right)\right\|_{2}^{2} ;
$$

其中, $P_{\mathrm{AN}}\left(\boldsymbol{A}_{2 \mathrm{DL}}^{i}\right)=\boldsymbol{R}_{3 \mathrm{D}}^{i} / \boldsymbol{R}_{2 \mathrm{DL}}^{i} \times\left(\boldsymbol{R}_{2 \mathrm{DL}}^{i}\right), \boldsymbol{R}_{3 \mathrm{D}}^{i}$ 和 $\boldsymbol{R}_{2 \mathrm{DL}}^{i}$ 表示 $\boldsymbol{f}_{3 \mathrm{D}}^{t}$ 和 $\boldsymbol{f}_{2 \mathrm{DL}}^{t}$ 各内角角度.

面积约束能量

$$
E_{\mathrm{Area}}=\sum_{i \in \mathrm{AR}}\left\|\boldsymbol{A}_{2 \mathrm{DL}}^{i}-P_{\mathrm{AR}}\left(\boldsymbol{A}_{2 \mathrm{DL}}^{i}\right)\right\|_{2}^{2} ;
$$

其中, $P_{\mathrm{AR}}\left(\boldsymbol{A}_{2 \mathrm{DL}}^{i}\right)=\boldsymbol{A}_{3 \mathrm{D}}^{i} / \boldsymbol{A}_{2 \mathrm{DL}}^{i} \times\left(\boldsymbol{A}_{2 \mathrm{DL}}^{i}\right), \boldsymbol{A}_{3 \mathrm{D}}^{i}$ 和 $\boldsymbol{A}_{2 \mathrm{DL}}^{i}$ 表示 $\boldsymbol{f}_{3 \mathrm{D}}^{t}$ 和 $\boldsymbol{f}_{2 \mathrm{DL}}^{t}$ 面积.
部分模型约束能迭代逼近值如表 4 所示，显 然，文献[4]相比仅在面积约束能量中得到较好的 结果, 而本文平面布局方法各项约束能量的最终 数值均为 0 , 即二维折痕图在平面布局后与原三维 模型保持一致, 不存在拉伸、裁剪、挤压等变形, 本 文平面布局方法取得了更好的逼近效果.

表 4 本文与文献[4]方法约束能量值对比

\begin{tabular}{lrrrrccc}
\hline \multirow{2}{*}{ 模型 } & \multicolumn{3}{c}{ 文献[4] } & & \multicolumn{3}{c}{ 本文 } \\
\cline { 2 - 4 } \cline { 6 - 8 } & $E_{\text {Area }}$ & $E_{\text {Length }}$ & $E_{\text {Angle }}$ & & $E_{\text {Area }}$ & $E_{\text {Length }}$ & $E_{\text {Angle }}$ \\
\hline Gaussian & 0.180 & 64.293 & 80.807 & 0 & 0 & 0 \\
Face & 0.223 & 262.458 & 441.949 & 0 & 0 & 0 \\
Ball & 0.218 & 90.846 & 649.347 & 0 & 0 & 0 \\
Leaf & 0.000 & 53.448 & 844.913 & 0 & 0 & 0 \\
Car & 0.024 & 449.623 & 649.724 & 0 & 0 & 0 \\
\hline
\end{tabular}

\section{5 折痕图有效性}

三维网格模型折叠过程中的刚性及折痕图的 稳定性可以通过基于目标模型同胚映射到圆盘的 稳定性来衡量 ${ }^{[2]}$. 本文方法对实验模型的自由度见 表 1 中的 $I$ 栏.

如图 16 所示, 折痕图的稳定性由趋势线(虚 线)表示, 即总体线性拟合的斜率; 自由度随着三 角面片数量和边数的增加而增大. 浅棕色曲线和 浅蓝色曲线为文献[2]方法的数据曲线, 仅可应用 在多边形数目少于 400 的模型, 其趋势线的斜率较 小, 稳定性较弱. 深棕色曲线和深蓝色曲线为本文

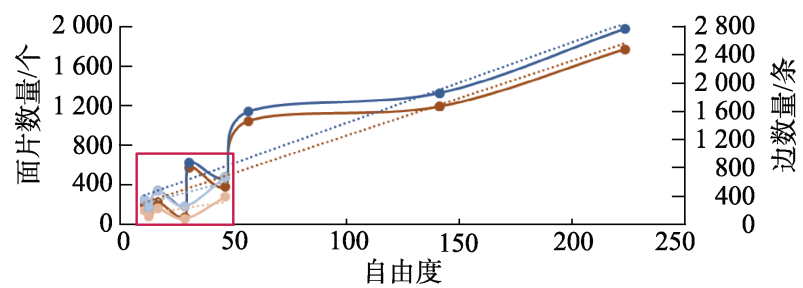

a. 本文与文献[2]稳定性对比

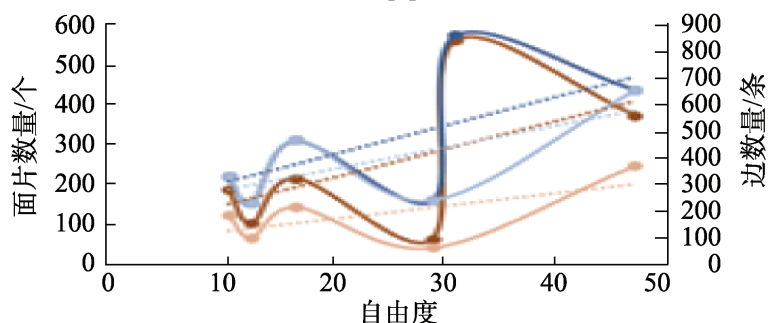

b. 图a红色区域局部放大

- 本文边自由度曲线;

$\longrightarrow$ 文献[2]边自由度曲线;

- - 本文面片自由度曲线; 本文边曲线线性趋势;

- 文献[2]边曲线线性趋势; 文献[2]面片自由度曲线； 本文面片曲线线性趋势; 文献[2]面片曲线线性趋势

图 16 折痕图的结构稳定性 
方法的数据曲线, 适用于面片数更多、边数更多的 复杂模型，其趋势线的斜率更大，稳定性更强.

验证本文折痕图有效性的另一种方法是计算 输人模型的表面积与待折叠正方形纸的表面积之 比，即有效值 $E=S_{3 \mathrm{D}} / S_{\text {Crease }}$ ，其值均大于 0.1 ; 当 比值越趋近于 0 时，实验结果的有效性越高. 表 5 所示为各模型使用不同参数化方法获得的有效值, 一般模型有效值的取值为 $0.1 \sim 0.4$. 本文实验结果 表明，以 LSCM 计算的折痕图有效值大多超过 0.4; 以 MEAN 计算 Leaf 折痕图的有效值最低，趋近于 0 ; 而以 ARAP 计算的折痕图有效值均为 $0.1 \sim 0.4$, 表明本文 ARAP 有效性最高.

表 5 ARAP, LSCM, MEAN 方法中不同模型的有效值

\begin{tabular}{lccc}
\hline \multicolumn{1}{c}{ 模型 } & ARAP $^{[3]}$ & LSCM $^{[13]}$ & MEAN $^{[14]}$ \\
\hline Gaussian & 0.1310 & & 0.1711 \\
Face & 0.3491 & 0.7345 & 2.1536 \\
Ball & 0.1296 & 0.3712 & 0.1297 \\
Leaf & 0.0687 & 0.0396 & 0.0001 \\
Car & 2.0000 & 31.2108 & \\
\hline
\end{tabular}

\section{5 结 语}

本文基于 ARAP 局部参数化预处理以及刚性 的均匀平面布局，实现了三角网格模型 VTM 和 ETM 的设计. 与其他相关工作对比, 通过调整平 移参数, 本文方法的平面布局误差和稳定性较优.

本文方法目前仅适用于开放边界的三角网格 模型，在处理具有复杂表面多边形和具有复杂边 界的三维网格模型效果较差，需要重新考虑优化 平面布局问题; 在处理封闭的三维网格模型时，需 要在初始化阶段之前进行分割预处理.下一步工 作将考虑基于形状语义的自动分割算法以及针对 封闭网格模型的最优平面布局算法.

\section{参考文献(References):}

[1] Schmidt P, Born J, Campen M, et al. Distortion-minimizing injective maps between surfaces[J]. ACM Transactions on Graphics, 2019, 38(6): Article No.156

[2] Tachi T. Origamizing polyhedral surfaces[J]. IEEE Transactions on Visualization and Computer Graphics, 2010, 16(2): 298-311

[3] Liu L G, Zhang L, Xu Y, et al. A local/global approach to mesh parameterization $[\mathrm{C}] / /$ Proceedings of the Symposium on Geometry Processing. Aire-la-Ville: Eurographics Association Press, 2008: 1495-1504

[4] Konaković-Luković M, Panetta J, Crane K, et al. Rapid deployment of curved surfaces via programmable auxetics[J]. ACM Transactions on Graphics, 2018, 37(4): Article No.106
[5] Tachi T. Freeform origami tessellations by generalizing Resch's patterns $[\mathrm{C}] / /$ Proceedings of the ASME 2013 International Design Engineering Technical Conferences and Computers and Information in Engineering Conference. New York: ASME Press, 2013: Article No.12326.

[6] Zhu L F, Igarashi T, Mitani J. Soft folding[J]. Computer Graphics Forum, 2013, 32(7): 167-176

[7] Zhao Y, Sugiura Y, Tada M, et al. InsTangible: a tangible user interface combining pop-up cards with conductive ink printing[C] //Proceedings of International Conference on Entertainment Computing. Heidelberg: Springer, 2017: 72-80

[8] Miyashita S, Guitron S, Li S G, et al. Robotic metamorphosis by origami exoskeletons[J]. Science Robotics, 2017, 2(10): 4369: $1-6$

[9] Zhao Y, Kanamori Y, Mitani J. Design and motion analysis of axisymmetric 3D origami with generic six-crease bases[J]. Computer Aided Geometric Design, 2018: 59: 86-97

[10] Li S G, Stampfli J J, Xu H J, et al. A vacuum-driven origami "magic-ball" soft gripper[C] //Proceedings of the IEEE International Conference on Robotics and Automation. Los Alamitos: IEEE Computer Society Press, 2019: 7401-7408

[11] Sheffer A. Spanning tree seams for reducing parameterization distortion of triangulated surfaces[C]//Proceedings of the Shape Modeling International. Los Alamitos: IEEE Computer Society Press, 2002: 61-66

[12] Sheffer A, Lévy B, Mogilnitsky M, et al. ABF++: fast and robust angle based flattening[J]. ACM Transactions on Graphics, 2005, 24(2): 311-330

[13] Lévy B, Petitjean S, Ray N, et al. Least squares conformal maps for automatic texture atlas generation[J]. ACM Transactions on Graphics, 2002, 21(3): 362-371

[14] Wang H, Sidorov K A, Sandilands P, et al. Harmonic parameterization by electrostatics[J]. ACM Transactions on Graphics, 2013, 32(5): Article No.155

[15] Sawhney R, Crane K. Boundary first flattening[J]. ACM Transactions on Graphics, 2018, 37(1): Article No.5

[16] Du Xingyi, Yan Dongming, Ye Juntao, et al. Quad mesh generation via field-aligned centroidal Voronoi tessellation[J]. Journal of Computer-Aided Design \& Computer Graphics, 2018, 30(5): 20-27(in Chinese)

(杜兴逸, 严冬明, 叶军涛, 等. 基于场对齐质心 Voronoi 划 分的四边网格生成[J]. 计算机辅助设计与图形学学报, 2018, 30(5): 20-27)

[17] Liu L G, Ye C Y, Ni R Q, et al. Progressive parameterizations[J]. ACM Transactions on Graphics, 2018, 37(4): Article No.41

[18] Soliman Y, Slepčev D, Crane K. Optimal cone singularities for conformal flattening $[\mathrm{J}]$. ACM Transactions on Graphics, 2018, 37(4): Article No.105

[19] Chen Y, Peng R, You Z. Origami of thick panels[J]. Science, 2015, 349(6246): 396-400

[20] Chen Y, Feng H J, Ma J Y, et al. Symmetric waterbomb origami[J]. Proceedings of the Royal Society A, 2016, 472(2190): 20150846

[21] Guseinov R, Miguel E, Bickel B. CurveUps: shaping objects from flat plates with tension-actuated curvature[J]. ACM Transactions on Graphics, 2017, 36(4): Article No.64

[22] Li S G, Vogt D M, Rus D, et al. Fluid-driven origami-inspired artificial muscles $[\mathrm{J}]$. Proceedings of the National Academy of Sciences of the United States of America, 2017, 114(50): 13132-13137

[23] Wang Z Q, Song P, Isvoranu F, et al. Design and structural optimization of topological interlocking assemblies[J]. ACM Transactions on Graphics, 2019, 38(6): Article No.193 